\title{
Production of p-nuclei in ejecta from a neutrino-driven, aspherical supernova
}

\section{Shin-ichiro Fujimoto*}

Kumamoto National College of Technology, Kumamoto 861-1102, Japan

E-mail: fujimoto@ec.knct.ac.jp

\section{Masaomi Ono,}

Yukawa Institute for Theoretical Physics, Kyoto University, Kyoto, 606-8502, Japan

\section{Masa-aki Hashimoto, Yukihiro Kikuchi,}

Department of Physics, School of Sciences, Kyushu University, Fukuoka 810-8560, Japan

\section{Kei Kotake}

Division of Theoretical Astronomy, National Astronomical Observatory Japan, 2-21-1, Osawa,

Mitaka, Tokyo, 181-8588, Japan

\begin{abstract}
We have examined explosive nucleosynthesis of $p$-nuclei during a neutrino-driven, aspherical supernova aided by both convection and standing accretion shock instability, based on a twodimensional hydrodynamic simulation of the explosion of a $15 M_{\odot}$ star, with an explosion energy of $\sim 10^{51} \mathrm{ergs}$. We find that $p$-nuclei are mainly produced by $\gamma$-processes, and that the nuclei lighter than ${ }^{92}$ Mo are abundantly synthesized in slightly neutron-rich bubbles with electron fractions of $Y_{e} \leq 0.48$, although masses of the bubbles are highly uncertain in the current hydrodynamic simulation. Abundances of the $p$-nuclei are comparable to those in a spherical model, and nuclei, ${ }^{94} \mathrm{Mo},{ }^{96} \mathrm{Ru}$, and ${ }^{98} \mathrm{Ru}$, are underproduced compared with the solar system for our aspherical models, as in a spherical model. ${ }^{138} \mathrm{La}$ and ${ }^{180} \mathrm{Ta}$, which are underproduced in the model are abundantly produced by charged current neutrino-interactions with ${ }^{138} \mathrm{Ba}$ and ${ }^{180} \mathrm{Hf}$, respectively.
\end{abstract}

XII International Symposium on Nuclei in the Cosmos,

August 5-12, 2012

Cairns, Australia

* Speaker. 


\section{Introduction}

There exist 35 stable nuclei on the proton-rich side of the s-process path with mass number $\mathrm{A} \geq 74$ referred to as $p$-nuclei. Many production sites of the $p$-nuclei have been proposed (e.g. [1] and references therein). The most promising site is the oxygen/neon layers during core-collapse supernova (SN) explosion [2]. The $p$-nuclei are synthesized by the photodisintegrations of $s$-process seeds produced in the layers during the core helium burning in the progenitor. An initial series of photo-disintegration reactions of type $(\gamma, \mathrm{n})$ is later followed by a mix of photo-disintegration reactions that also include $(\gamma, \mathrm{p})$ and/or $(\gamma, \alpha)$ reactions. The production of $p$-nuclei by photodisintegration is referred to as a $\gamma$-process. Extensive investigations of the $\gamma$-process in core-collapse $\mathrm{SNe}$ are performed by [2], in which the overall abundance profile of $p$-nuclei in the solar system is reproduced in ejecta in the $\mathrm{O} / \mathrm{Ne}$ layers during $\mathrm{SNe}$. The model, however, has a conspicuous shortcoming of underproduction of ${ }^{92,94} \mathrm{Mo},{ }^{96,98} \mathrm{Ru}$, and ${ }^{138} \mathrm{La}$. Abundant production of such $p$ nuclei due to neutrino processes has been found by [3, 4]; The yield of ${ }^{138} \mathrm{La}$ in the $\mathrm{O} / \mathrm{Ne}$ layers is increased via neutrino interactions but those of $\mathrm{Mo}$ and $\mathrm{Ru}$ are not appreciably changed [4].

The explosion mechanism of core collapse SNe is still not clearly understood. Multi-dimensional effects are recognized to be important for SN explosion, in particular for a progenitor heavier than about $11 M_{\odot}$ in its main sequence phase [5, 6]. Standing accretion shock instability (SASI) is a reliable candidate to initiate bipolar oscillations of a stalled shock. Recent two- or three-dimensional (2D or 3D) simulations of stellar core collapse showed that delayed neutrino-driven mechanism aided by both SASI and convection is likely to cause an aspherical explosion of non- or slowlyrotating massive stars [6, 7, 8].

In our previous study [9], we investigated explosive nucleosynthesis during a delayed neutrinodriven SN aided by SASI and convection, in particular from $\mathrm{C}$ to $\mathrm{Zn}$, based on 2D hydrodynamic simulations of the explosion of a $15 M_{\odot}$ star with initial solar metallicity using a hydrodynamic code with a simplified neutrino transport scheme. We showed that aspherical abundance distributions, which are observed in nearby core-collapse SN remnants, such as Cas A and Cygnus loop, are obtained despite of a non-rotating, spherical progenitor, due to the growth of a low-mode SASI. The abundance pattern from $\mathrm{C}$ to $\mathrm{Zn}$ of the aspherical SN ejecta is similar to that of the solar system, for models in which the explosion energies and the ${ }^{56} \mathrm{Ni}$ masses are evaluated to be $\simeq 10^{51} \mathrm{ergs}$ and $0.05-0.06 M_{\odot}$, respectively, and the estimated masses of a neutron star are comparable with the baryonic mass of neutron stars observed in binary neutron stars.

In the present study, we examine explosive nucleosynthesis of $p$-nuclei during a delayed neutrino-driven SN based on 2D hydrodynamic simulations of the explosion of a $15 M_{\odot}$ star with initial solar metallicity for a model with an explosion energy of $\simeq 10^{51}$ ergs. In $\S 2$ we briefly describe our numerical code for hydrodynamic calculation of $\mathrm{SN}$, the initial conditions of the progenitor star, and the properties of the aspherical explosion. In $\S 3$, we present a large nuclear reaction network and the abundances of $p$-nuclei in the ejecta. Finally we will summarize our results in $\S 4$.

\section{Hydrodynamic code and hydrodynamics of supernova explosion}

To calculate the structure and evolution of the collapsing and exploding star, we solve the 
Newtonian hydrodynamic equations,

$$
\begin{gathered}
\frac{D \rho}{D t}+\rho \nabla \cdot \mathbf{v}=0 \\
\rho \frac{D \mathbf{v}}{D t}=-\nabla P-\rho \nabla\left(\Phi+\Phi_{c}\right) \\
\rho \frac{d}{d t}\left(\frac{e}{\rho}\right)=-P \nabla \cdot \mathbf{v}+Q_{\mathrm{E}} \\
\frac{D Y_{e}}{D t}=Q_{\mathrm{N}}
\end{gathered}
$$

where $\rho, P, \mathbf{v}, e$, and $Y_{e}$, are the mass density, the pressure the fluid velocity, the internal energy density, and the electron fraction, respectively. We denote the Lagrangian derivative as $D / D t$. The gravitational potential of the fluid and of the central object with mass of $M_{\mathrm{pNS}}, \Phi$ and $\Phi_{c}$, are evaluated using

$$
\triangle \Phi=4 \pi G \rho
$$

and

$$
\Phi_{c}=-\frac{G M_{\mathrm{pNS}}}{r},
$$

where $G$ is the gravitational constant. We note that the mass of the proto-neutron star (PNS), $M_{\mathrm{pNS}}$, continuously increases due to mass accretion through the inner boundary. Although a small fraction of mass energy of PNS decreases due to neutrino emission, the decreased mass energy is less than $10 \%$ of an increased mass energy through the mass accretion at $<0.4 \mathrm{~s}$, since the mass accretion rate is greater than $2.5 M_{\odot} \mathrm{s}^{-1}$.

$Q_{\mathrm{E}}$ and $Q_{\mathrm{N}}$ are the source terms that describe the rate of change per unit volume in equations (2.3) and (2.4), respectively, and are summarized in Appendix A and B in [9]. We take into account absorption of electron and anti-electron neutrinos as well as neutrino emission due to electron and positron captures, electron-positron pair annihilation, nucleon-nucleon bremsstrahlung, and plasmon-decays. We assume that the fluid is axisymmetric and that neutrinos are isotropically emitted from the neutrino spheres with given luminosities and with the Fermi-Dirac distribution of given temperatures [10]. The numerical code for the hydrodynamic calculations employed in this paper is based on the ZEUS-2D code [10, 11], and has been used to analyze nucleosynthesis in a neutrino-driven $\mathrm{SN}$ of a $15 M_{\odot}$ solar metallicity star in our previous work [9].

We have performed a simulation of the explosion of the $15 M_{\odot}$ star [9] for a model with an electron-neutrino luminosity of $L_{v_{e}}=4.5 \times 10^{52} \mathrm{erg} \mathrm{s}^{-1}$ from the core collapse to $5 \mathrm{~s}$ after core bounce, when a shock front has reached a layer with $r=30,000 \mathrm{~km}$ in all directions. We set $L_{\bar{v}_{e}}=L_{v_{e}}$ and $L_{v_{x}}=0.5 L_{v_{e}}$, where $L_{\bar{v}_{e}}$ and $L_{v_{x}}$ are the luminosities of anti-electron and other-types of neutrinos $\left(\mu, \tau\right.$, anti- $\mu$, and anti- $\tau$ ), respectively. We use neutrino temperatures $T_{v_{e}}, T_{\bar{v}_{e}}$, and $T_{v_{x}}$ of $4 \mathrm{MeV}, 5 \mathrm{MeV}$, and $10 \mathrm{MeV}$, respectively [10].

In our previous work [9], we showed that the shape of shock front is quasi-spherical, although the distribution of neutrino heated ejecta is highly aspherical in the model. In addition, we found that the explosion energy is $\sim 10^{51} \mathrm{ergs}$ and that abundance pattern $(\mathrm{A}<60)$ of the ejecta is similar to that of the solar system. 


\section{Abundances of $p$-nuclei}

In order to calculate chemical composition of the SN ejecta, we adopt a tracer particle method to obtain the Lagrangian evolution of the physical quantities from the Eulerian evolution obtained from our simulation, as in the previous work [9]. We initially place 3,000 particles from 300 to $30,000 \mathrm{~km}$, or from the iron-core to O-rich layers, and 30 particles in a layer with a fixed interval on $\theta$. The mass of a particle in the layer is weighted to the mass of the layer. We have confirmed that the estimated energies and masses of the ejecta with the 3,000 particles equal to those with 6,000 particles within $1 \%$ and abundance profiles are similar [9]. We note that the minimum mass of the particles is $\sim 10^{-4} M_{\odot}$. We find that more than about 2,700 particles are ejected due to the aspherical explosion. The initial abundances of the particles are set to be those of the star at their initial location just before core collapse [12], in which 1400 nuclei are taken into account. Next, we calculate abundances and masses of the supernova ejecta. Ejecta that is located on the inner region of the star $\left(r_{\mathrm{cc}} \leq 30,000 \mathrm{~km}\right)$ before core collapse has enough high maximum temperatures for elements heavier than $\mathrm{C}$ to burn explosively. Here $r_{\mathrm{cc}}$ is the radius at core collapse. We then follow the abundance evolution of the ejecta from the inner region using a nuclear reaction network which includes 1989 nuclide from neutron and proton to Bi [13]. The composition of ejecta from the outer region $\left(r_{\mathrm{cc}}>30,000 \mathrm{~km}\right)$ is set to be that before the core collapse [12]. Moreover, when the temperature of the ejecta becomes greater than $9 \times 10^{9} \mathrm{~K}$, we set the chemical composition of the ejecta to be in nuclear statistical equilibrium (NSE), whose abundances are expressed with simple analytical expressions, specified by the density, temperature and electron fraction.
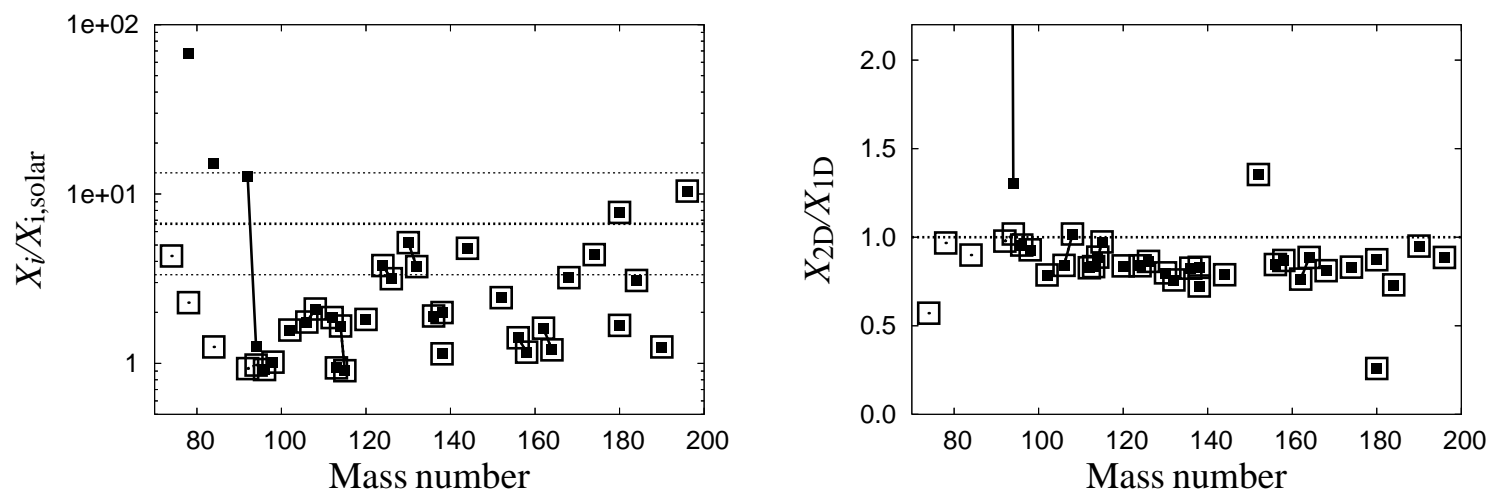

Figure 1: Overproduction factors of $p$-nuclei (left panel) and abundance ratios of $p$-nuclei between our 2D model and 1D spherical model (right panel) as a function of mass number $Z$ for all ejecta (filled squares) and ejecta with electron fraction $Y_{e}>0.48$ (open squares). The abundances of our 2D model are very similar to those in the 1D model [12], except for $p$-nuclei lighter than ${ }^{92} \mathrm{Mo}$, which are abundantly produced in slightly neutron-rich ejecta $0.46<Y_{e}<0.48$.

Figure 1] shows overproduction factors (= ratios of abundances to those in the solar system) and abundance ratios of $p$-nuclei as a function of mass number $Z$. The abundances of our $2 \mathrm{D}$ model are very similar to those in the 1D model [12], except for $p$-nuclei lighter than ${ }^{92} \mathrm{Mo}$. This is because the shape of shock front is quasi-spherical, although the distribution of neutrino heated ejecta is aspherical [9]. As in a spherical model [2], ${ }^{94} \mathrm{Mo},{ }^{96} \mathrm{Ru},{ }^{98} \mathrm{Ru},{ }^{113} \mathrm{In},{ }^{115} \mathrm{Sn},{ }^{138} \mathrm{La}$, and 
${ }^{180} \mathrm{Ta}$ are underproduced compared to the solar system for our aspherical models. Moreover, $p$ nuclei lighter than ${ }^{92} \mathrm{Mo}$ are found to be abundantly synthesized in slightly neutron-rich ejecta $\left(Y_{e} \sim 0.46-0.48\right)[14]$.
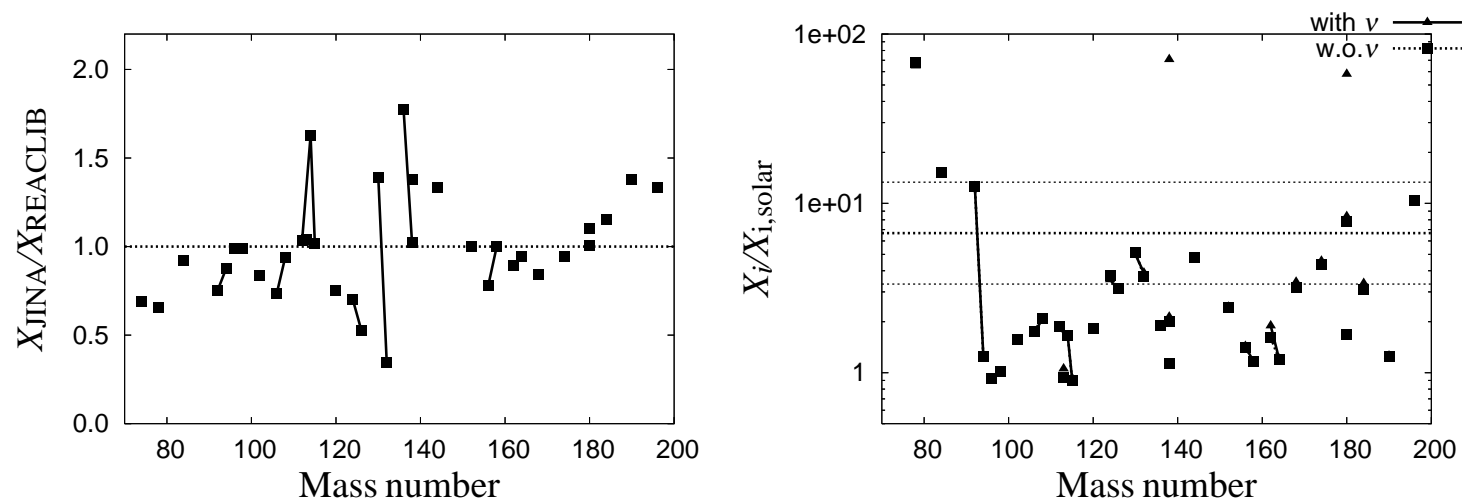

Figure 2: Abundance ratios of $p$-nuclei between ejecta evaluated with two reaction networks (left panel), in which REACLIB and JINA REACLIB Ver. 1 [15] compilations of reaction rates are employed, and overproduction factors of $p$-nuclei (right panel) with (filled triangles) and without (filled squares) neutrinointeractions related to the production of ${ }^{138} \mathrm{La}$ and ${ }^{180} \mathrm{Ta}$ as a function of mass number $Z$ for all ejecta.

Figure 2 shows abundance ratios of $p$-nuclei of ejecta evaluated with two reaction networks and overproduction factors of $p$-nuclei with and without neutrino-interactions related to the production of ${ }^{138} \mathrm{La}$ and ${ }^{180} \mathrm{Ta}$ as a function of $A$ for all ejecta. We find that a change in the reaction rates results in a minor effect, less than a factor of three, in abundances of $p$-nuclei; ${ }^{114} \mathrm{Sn}$ and ${ }^{136} \mathrm{Ce}$ are enhanced by $\sim 62.6 \%$ and $\sim 87.3 \%$, respectively, but ${ }^{132}$ Ba decreases by $\sim 189 \%$. The differences could be due to the experimental $\gamma$-induced reaction rates in JINA REACLIB.

Effects of neutrino interactions on the productions of ${ }^{138} \mathrm{La}$ and ${ }^{180} \mathrm{Ta}$ have been examined [4. 16] with spherical explosion models, and ${ }^{138} \mathrm{La}$ and ${ }^{180} \mathrm{Ta}$ are shown to be enhanced due to charged-current reactions of ${ }^{138} \mathrm{Ba}$ and ${ }^{180} \mathrm{Hf}$. In our 2D models, ${ }^{138} \mathrm{La}$ and ${ }^{180} \mathrm{Ta}$ are significantly overproduced compared with those in the solar system as well as in the previous works [4, 16]. The cross sections of the absorptions taken from [17] is comparable to those in [16]. The neutrino luminosity adopted in our models, $L_{v_{e}}=4.5 \times 10^{52} \mathrm{erg} \mathrm{s}^{-1}$, is simply assumed to be constant in time. Therefore, at late time, the luminosity is higher than that in [16], in which $L_{v_{e}}$ is initially comparable to ours $\left(5 \times 10^{52} \mathrm{erg} \mathrm{s}^{-1}\right)$, but is assumed to decay exponentially on a time-scale of $3 \mathrm{~s}$. Hence, our abundances of ${ }^{138} \mathrm{La}$ and ${ }^{180} \mathrm{Ta}$ could be over-estimated in our $2 \mathrm{D}$ model.

\section{Summary}

We examined explosive nucleosynthesis of $p$-nuclei during a neutrino-driven, aspherical supernova aided by both convection and standing accretion shock instability based on 2D hydrodynamic simulations of the explosion of a $15 M_{\odot}$ star with an explosion energy of $\sim 10^{51} \mathrm{ergs}$, producing an abundance pattern $(\mathrm{A}<60)$ of the ejecta similar to that of the solar system. The abundances of $p$ nuclei in the SN ejecta are calculated using a large nuclear reaction network. We find that $p$-nuclei are mainly produced by $\gamma$-processes, and that the nuclei lighter than ${ }^{92} \mathrm{Mo}$ are abundantly synthe- 
sized in slightly neutron-rich bubbles with electron fractions of $0.46 \leq Y_{e} \leq 0.48$, whose masses are highly uncertain in the current hydrodynamic simulations. The abundances of the $p$-nuclei are comparable to those of a spherical model, except for the $p$-nuclei lighter than ${ }^{92} \mathrm{Mo}$. The nuclei ${ }^{94} \mathrm{Mo},{ }^{96} \mathrm{Ru}$, and ${ }^{98} \mathrm{Ru}$ are underproduced compared to the solar system for our aspherical models, similar to the spherical models [2, 12]. ${ }^{138} \mathrm{La}$, and ${ }^{180} \mathrm{Ta}$, which have been confirmed to be enhanced in the spherical models [4, 16], are abundantly produced by charged-current neutrino-interactions on ${ }^{138} \mathrm{Ba}$ and ${ }^{180} \mathrm{Hf}$, respectively.

\section{Acknowledgments}

This work was supported in part by a Grant-in-Aid for Scientific Research from the Ministry of Education, Culture, Sports, Science and Technology of Japan (No. 22540297).

\section{References}

[1] Arnould, M., \& Goriely, S. 2003, Physics Report, 384, 1

[2] Rayet, M., Arnould, M., Hashimoto, M., Prantzos, N., \& Nomoto, K.1995, A\&A 298, 517

[3] Woosley, S. E., Hartmann, D. H., Hoffman, R. D.,\& Haxton, W. C. 1990, ApJ , 356, 272

[4] Goriely, S., Arnould, M., Borzov, I., \& Rayet, M.2001, A\&A 375, 35

[5] Kitaura, F. S., Janka, H.-T., \& Hillebrandt, W. 2006, A\&A 450, 345

[6] Marek, A., \& Janka, H.-T.2009, ApJ 694, 664

[7] Takiwaki, T., Kotake, K., \& Suwa, Y. 2012, ApJ , 749, 98

[8] Müller, B., Janka, H.-T., \& Heger, A. 2012, ApJ , 761, 72

[9] Fujimoto, S., Kotake, K., Hashimoto, M., Ono, M., \& Ohnishi, N. 2011, ApJ 738, 61

[10] Ohnishi, N., Kotake, K., \& Yamada, S. 2006, ApJ , 641, 1018

[11] Stone, J. M., \& Norman, M. L. 1992, ApJS , 80, 791

[12] Rauscher, T., Heger, A., Hoffman, R. D., \& Woosley, S. E. 2002, ApJ , 576, 323

[13] Fujimoto, S., Hashimoto, M., Koike, O., Arai, K., \& Matsuba, R. 2003, ApJ , 585, 418

[14] Hoffman, R. D., Woosley, S. E., Fuller, G. M., \& Meyer, B. S. 1996, ApJ 460, 478

[15] Cyburt, R. H., et al., 2010, ApJS, 189, 240

[16] Heger, A., Kolbe, E., Haxton, W. C., et al. 2005, Physics Letters B, 606, 258

[17] Byelikov, A., Adachi, T., Fujita, H., et al. 2007, Physical Review Letters, 98, 082501 